\title{
Guidance to post-mortem collection and storage of biological specimens for the diagnosis of Covid-19 infection
}

\author{
Francesco d'Aleo, ${ }^{1,2}$ Milena Arghittu, ${ }^{2,3}$ Claudio Bandi, ${ }^{2,4}$ Marco Conte, ${ }^{1}$ Roberta Creti, ${ }^{2,5}$ \\ Claudio Farina ${ }^{2,6}$ \\ ${ }^{1}$ Microbiology and Virology Unit, Grande Ospedale Metropolitano, Reggio Calabria; ${ }^{2}$ GLAMIFO - Study Group on \\ Forensic Microbiology, AMCLI, Milan; ${ }^{3}$ Analysis Laboratory, Vizzolo Predabissi Hospital, ASST Melegnano \\ Martesana; ${ }^{4}$ Department of Sciences, University of Milan; ${ }^{5}$ Department of Infectious Diseases, National Insititute of \\ Health, Rome; 'Microbiology and Virology Unit, ASST Papa Giovanni XXIII Hospital, Bergamo, Italy
}

\begin{abstract}
Summary
The following document is based on the current knowledge of SARS-CoV-2 and the related disease (COVID-19). The Italian Institute of Health (ISS) is monitoring the virus spread throughout the country through daily reports sent by individual regions and regional reference laboratories. The diagnosis of infection is based on the use of standardized and validated molecular tests for the search for viral RNA in different sets of biological samples, from patients suspected of having contracted the infection. This document provides a specific guidance for the collection and storage of biological samples from deceased persons. It also provides recommendations on safety practices to be adopted during both collection and handling of specimens, and during autopsy procedures. With regard autopsy, it is recommended to follow the procedures for the execution of diagnostic findings in patients died with SARS-CoV-2 infection issued by ISS Working Group on Causes of Death from COVID-19, published on March 27, 2020.
\end{abstract}

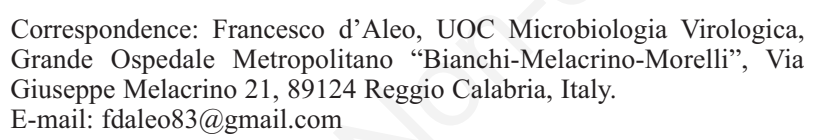

Key words: forensic microbiology; post-mortem collection; COVID-19.

Contributions: the authors contributed equally.

Conflict of interest: the authors declare no potential conflict of interest.

Funding: none.

Acknowledgements: The authors wish to thank Dr. Ersilia Fiscarelli for the English revision of the text.

Received for publication: 17 June 2020.

Accepted for publication: 23 July 2020.

${ }^{\circ}$ Copyright: the Author(s), 2020

Licensee PAGEPress, Italy

Microbiologia Medica 2020; 35:9191

doi:10.4081/mm.2020.9191

This article is distributed under the terms of the Creative Commons Attribution Noncommercial License (by-nc 4.0) which permits any noncommercial use, distribution, and reproduction in any medium, provided the original author(s) and source are credited.

\section{Specimen collection}

Since collection of upper respiratory tract material performed on corpse does not generate aerosols, it is sufficient to follow the standard precautions. It is necessary to wear the following disposable devices: three pairs of gloves having the outer and inner layer in latex and the middle in non-sterile and cut-resistant nitrile, waterproof and liquid-resistant long-sleeved gown; waterproof apron; plastic face protection and glasses; mask; boots or shoe covers and headgear. Following dressing and undressing procedures as indicated by the company and national protocols is mandatory. In the event of an autopsy, it is absolutely necessary to avoid using the oscillating saw and opt for manual shears. If the use of the oscillating saw cannot be deferred, it is necessary to have a vacuum system to contain the aerosol. Only one person can make the cut, even if manual, and it is imperative to minimize the personnel necessary for carrying out the sectoral practice.

It is recommended to perform the biological specimens from the upper respiratory tract and, if the autopsic examination is performed, the swabs from the lower respiratory tract should be tested as well. It is also recommended to use only flocked swabs preferably UTM (Universal Transport Medium for Viruses, Chlamydia, Mycoplasma and Ureaplasma); do not use calcium alginate or wooden rod swabs as they may contain substances that inhibit the polymerase chain reaction (PCR). The collected specimens must be individually recorded and stored, in compliance with the maintenance of the cold chain, in the fridge at a temperature of $2-8^{\circ} \mathrm{C}$.

If an autopsy is not performed, it is recommended to withdraw the following biological specimens:

nasopharyngeal swab; oropharyngeal swab within two hours of death. It is recommended not to put together the specimens as it may be necessary to use them for the research of other respiratory pathogens.

In order to perform the nasal swab, the following procedure should be followed: insert the swab shaft inside the right nostril parallel to the palate until it reaches the rear area of the nasopharynx. Rotate the swab and leave it in place for 15 seconds, so that it can absorb any secretions present. With the same swab repeat the same procedure in the left nostril. Remove the swab, break the loop inside the tube containing the transport medium, plug and label it.

For the execution of the oropharyngeal swab, after having opened the mouth wide, insert the swab behind both the tonsil pillars and the uvula taking care to avoid any contact with the tongue. Turn accurately the swab well and plug it as described above.

If an autopsy is performed, it is recommended to withdraw the 
following biological samples: nasopharyngeal swab; oropharyngeal swab within two hours of death; bronchial/lung swab (to be performed for each lung); fecal material. For the purpose of possible scientific investigation, it would be appropriate to take aspirates of intra-ocular fluid and bone marrow in order to search for SARS-Cov-2 RNA.

Proceed with the collection of the nasopharyngeal and oropharyngeal swab as described above.

For the execution of the bronchial swab: after removing the sternal plate and exposing the right lung, proceed with the incision and opening of the relative bronchi. Since viral particles are subject to focal destruction in patients with respiratory infection, it is necessary to buffer the main and segmental bronchi as much as possible. Insert the swab in contact with the internal surface of each bronchus, rotate and then insert it, breaking the loop, in the appropriate bottle containing the transport medium. Repeat the same procedure for the left lung.

The collected specimens must be labeled separately. Research of other pathogens through molecular biology methods is recommended.

We recommend, whenever possible, the collection of tissues. In the case of an autoptic examination, removal of tissue fragments of right and left lung parenchyma is recommended; pulmonary hilus with fragments of segmental and primary bronchi; trachea fragments. The tissues collected, that must be stored in $10 \%$ buffered formalin, should have a maximum thickness of 4-5 mm, in order to favor the optimal fixation.

\section{Specimens safety}

Specimens collected properly and labeled must be placed inside a primary container with a support (test-tube holder) which must be closed inside a secondary container in which an adequate amount of absorbent paper is inserted; if possible, it should be placed inside a sealable plastic bag for transport. It should be born in mind that personnel in charge of the biological material transport from the autopsy room to the laboratory must necessarily wear disposable gloves.

\section{References}

Centres for Disease Control and Prevention. Collection and Submission of Postmortem Specimens from Deceased Persons with Known or Suspected COVID-19, March 2020 (Interim Guidance). Available from: https://www.cdc.gov/ coronavirus/2019-ncov/hcp/guidance-postmortem-specimens.html

Fernández-Rodríguez A, Burton JL, Andreoletti L, et al. Postmortem microbiology in sudden death: sampling protocols proposed in different clinical settings. Clin Microbiol Infect 2019;25:570-9.

Hanley B, Lucas SB, Youd E, et al. Autopsy in suspect COVID-19 cases. J Clin Pathol 2020;73:239-42.

National Association of Medical Examiners (NAME). Covid-19: postmortem diagnostic and biosafety consideration. Am J Forensic Med Pathol 24 Apr. 2020.

Santurro A, Scopetti M, D'Errico S, Fineschi V. A technical report from the Italian SARS-CoV-2 outbreak. Postmortem sampling and autopsy investigation in cases of suspected or probable COVID-19. Forensic Sci Med Pathol 2020:1-6.

Sapino A, Facchetti F, Bonoldi E, et al. The autopsy debate during the COVID-19 emergency: the Italian experience. Virchows Arch 2020;476,:821-3. 